\title{
Importance of Biostatistics in Medical Researches
}

\author{
Tıbbi Araştırmalarda Biyoistatistiğin Önemi
}

\author{
Mehmet Ali SUNGUR
}

Today's research and researchers tend to reach conclusions with the help of data, ever-increasing in size. Statistical methods are essential to researchers, as they transform raw data into information and produce meaningful results. More information can be get from data if it is known how variable they are; in a way, a picture of the data can draw with the pieces of information. The most valuable information about complex causal relationships can be obtained with the help of various statistical methods. For these reasons, as in all fields of researches, physicians making researches in medical sciences need to benefit from biostatistics more and more frequently and intensively. The value of research is also related to its biostatistical value, besides its subject, being up-to-date, important, and useful results, etc. Researches should be planned and carried out following scientific principles. Compliance of a study with scientific principles is also closely related to the correct and conscious use of biostatistics methods and principles.

In every research conducted by obtaining and analyzing data, the results section is substantially based on the findings obtained with statistical methods. Therefore, the reliability and validity of the findings depend on the accuracy of the statistical methods and approaches used. The validity of the results of a research conducted without complying with the correct and appropriate biostatistical methods will be doubtful and the reliability will be questionable. In addition to data analysis, biostatistics should also be used in the main points of methodology, such as designing the research in line with the purpose, determining the sample size, and considering the measure of errors, etc. The role of biostatistics in medical research starts at the planning stage. Valid statistical inference, and therefore decision-making, depends on robust planning. Therefore, biostatistics is essential not only for determining and using the methods to analyze data but also for using and benefitting from correct and conscious statistical methods and practice for the planning stage and also methodology. If all stages in research are not well established and planned, failure may occur at any stage of the research or the whole research. In such a case, the cost, time, and effort spent can be wasted. Therefore, a large part of research time should be devoted to the planning stage. Adequate use of statistical methods and statistical analysis of data is only possible if the design of the research is statistically sound. If the planning stage is not satisfactorily resolved, inference with the data and results obtained will never be adequate. As a result, biostatistical methods should be used appropriately at every stage of research from planning to publication.

In relatively simple situations where data are analyzed at the basic level, it may be sufficient to calculate simple values such as means, standard deviations, standard errors, confidence intervals or to apply standard parametric or non-parametric tests. Despite being used widely, even these basic concepts and tests are sometimes misunderstood, even not understood, or misinterpreted by researchers with limited statistical knowledge. Also, these basic calculations and analyses are just the tip of the iceberg and are the preliminary stage of the data analysis. To extract the maximum information from the data, it is often necessary to use more complex statistical methods. More complex research models require advanced statistical methods, which require the establishment and testing of advanced statistical models to make inferences from the data obtained. Such advanced methods should be applied in such a way that it becomes clear what the result means, considering both the place and purpose of their use. One of the reasons for misuse or misunderstanding is that the no or limited knowledge about the assumptions required by statistical methods, even at a basic level. When the assumptions required by the tests are not met, the results may be partially or completely wrong, leading to inaccurate conclusions and interpretations. Biostatisticians understand these issues and are aware of how and when seriously misleading may occur when the assumptions are not fulfilled. Another reason is over-analysis. The large number of results and $\mathrm{p}$-values that occur due to reasons 
such as performing some analyzes even though it is not necessary or appropriate, or analyzing the data separately, can be even more confusing for researchers.

To be used and benefit from biostatistical methods and principles smoothly and effectively in the reporting and discussion phase, close cooperation with a biostatistics expert is needed, whether the researchers are professionals in the relevant field or physicians with sound statistical knowledge. Biostatisticians can give useful guidance on planning, analyzing, reporting, etc. When this cooperation is best provided, it will make a valuable contribution to the validity and reliability of the research results.

To sum up, as in all field of researches, physicians making researches in medical sciences needs to know and interpret statistical results, at least at a statistical literacy level. Knowledge at a basic level about biostatistics and statistical methods is invaluable for the assessment of findings, clinical decision-making, and critical evaluation of the implementation of findings into medical and clinical practice. Providing appropriate and adequate training in the understanding and application of statistical methods and their results in medicine is crucial.

To support this, manuscripts submitted to Duzce Medical Journal are also reviewed by a biostatistics expert during the peer-review process, and comments and recommendations are given in more thoroughness and detail to support and assist the authors in improving and revising their manuscript and ultimately lead to a publication of the highest quality as much as possible.

In addition, starting from this issue, we start to publish informative articles in the field of biostatistics. In this issue, we share with our readers an invited review titled "Development of Biostatistics: From Past to Future" by Karahan and Karaağaoğlu, experts in the field. This review only scratches the history and surface of biostatistics in medical researches. Nonetheless, hopefully, this review makes a stimulus for physicians to further develop and deepen their ability in the appropriate use, understanding, and interpreting correctly of statistical methods.

On behalf of Duzce Medical Journal, we would like to thank the authors for this review and hope it will be useful to our readers.

\author{
Mehmet Ali SUNGUR
}

Editor in Chief, Duzce Medical Journal Biostatistics, Duzce University, Duzce/Turkey orcid.org/0000-0001-5380-0819 